\section{BMJ Paediatrics Open}

\title{
Transforming training into practice with the conflict management framework: a mixed methods study
}

Oscar Lyons (D) ," Liz Forbat (D) ," Esse Menson, ${ }^{3}$ Julia C Chisholm, ${ }^{4}$ Kate Pryde (D) , ${ }^{5}$ Siobhan Conlin, ${ }^{6}$ Victoria Felton, ${ }^{7}$ Susanne Ingle, ${ }^{8}$ Celia McKenzie, ${ }^{6}$ Rohana Ramachandran, ${ }^{7}$ Charlotte Sayer, ${ }^{9}$ Carly Snowball, ${ }^{10}$ Emma Strachan-Gadsby, ${ }^{7}$ Natasha Tisovszky, ${ }^{11}$ Sarah Barclay ${ }^{3}$

\section{ABSTRACT}

Objective To implement and evaluate the use of the conflict management framework (CMF) in four tertiary UK paediatric services.

Design Mixed methods multisite evaluation including prospective pre and post intervention collection of conflict data alongside semistructured interviews.

Setting Eight inpatient or day care wards across four tertiary UK paediatric services.

Interventions The two-stage CMF was used in daily huddles to prompt the recognition and management of conflict.

Results Conflicts were recorded for a total of 67 weeks before and 141 weeks after implementation of the CMF across the four sites. 1000 episodes of conflict involving 324 patients/families across the four sites were recorded. After implementation of the CMF, time spent managing episodes of conflict around the care of a patient was decreased by $24 \%(p<0.001$ ) (from 73 min to $55 \mathrm{~min}$ ) and the estimated cost of this staff time decreased by $20 \%$ ( $p<0.02$ ) (from £26 to £21 sterling per episode of conflict). This reduction occurred despite conflict episodes after implementation of the CMF having similar severity to those before implementation. Semistructured interviews highlighted the importance of broad multidisciplinary leadership and training to embed a culture of proactive and collaborative conflict management.

Conclusions The CMF offers an effective adjunct to conflict management training, reducing time spent managing conflict and the associated staff costs.

\section{INTRODUCTION}

Conflict is a complex phenomenon characterised by the experience of negative emotional reactions to perceived disagreements between two or more parties. ${ }^{1}$ Conflict is widely seen to be inevitable in healthcare settings. ${ }^{23}$ Without appropriate and timely management, conflict can result in harmful consequences for hospitals (legal costs, staff costs, increased staff turnover, decreased productivity), for staff (burnout, poor team cohesion, poor well-being) and most importantly for patients (including healthcare

\section{What is known about the subject?}

$\Rightarrow$ Conflict poses a considerable psychological and financial burden on clinical teams, patients and families.

$\Rightarrow$ Conflict management training has been shown to improve outcomes.

$\Rightarrow$ There is a need for improved structural support for conflict management.

\section{What this study adds?}

$\Rightarrow$ The conflict management framework was demonstrated to improve conflict management.

$\Rightarrow$ Use of the framework resulted in decreased time spent on conflict management and the staff cost of conflict.

$\Rightarrow$ Effective implementation requires strong crossdisciplinary conflict training and leadership to challenge cultural tolerance of conflict.

errors, poor therapeutic relationships and worse prognosis). ${ }^{14-9}$

Conflict management training has been shown to be effective in reducing the impact of conflict on hospitals, staff and patients/ families. $^{1-47810}$ To facilitate the transfer of learning from training into practice, there have been calls for development and evaluation of structured conflict management processes. $^{111-14}$

The conflict management framework (CMF) developed by the Medical Mediation Foundation (MMF) ${ }^{15}$ for use in paediatric settings is one of the few formalised approaches to managing healthcare conflict. The CMF has shown promise in reducing the frequency of conflicts and in improving staff self-reported scores of burnout in one Australian hospital. ${ }^{14}$

Building on previous work on recognising and managing conflict, this pilot project was 
Table 1 Site and staff demographics and data collection details

\begin{tabular}{|c|c|c|c|c|}
\hline & Site A & Site B & Site C & Site D \\
\hline \multicolumn{5}{|l|}{ Hospital demographics } \\
\hline Median patients per site (IQR) & $39(36-43)$ & $105(94-114)$ & $17(16-18)$ & $37(32-40)$ \\
\hline Median patient age (range) & 9 months (0-17 years) & 8 years $(1-24$ years $)$ & 20 months ( $0-17$ years) & 9 months ( $0-17$ years) \\
\hline Number of clinical areas & 2 & 4 & 1 & 1 \\
\hline \multicolumn{5}{|c|}{ Conflict management training demographics } \\
\hline Number of training sessions & 8 & 5 & 2 & 1 \\
\hline Number of staff trained & 133 & 101 & 17 & 14 \\
\hline Gender of staff trained (\%, female) & $89 \%$ & $94 \%$ & $89 \%$ & $86 \%$ \\
\hline Doctors & $23 \%$ & $17 \%$ & $36 \%$ & \\
\hline Consultant grade & $20 \%$ & $16 \%$ & $24 \%$ & \\
\hline Non-consultant grade & $2 \%$ & $1 \%$ & $12 \%$ & \\
\hline Nurses & $72 \%$ & $71 \%$ & $52 \%$ & $100 \%$ \\
\hline Matrons & $1 \%$ & $1 \%$ & 0 & \\
\hline Senior nurses* & $26 \%$ & $22 \%$ & $34 \%$ & \\
\hline Junior nurses & $45 \%$ & $48 \%$ & $18 \%$ & \\
\hline Allied health professionals & $5 \%$ & $12 \%$ & $12 \%$ & \\
\hline Therapists $\dagger$ & $2 \%$ & $8 \%$ & 0 & \\
\hline Other & $3 \%$ & $4 \%$ & $12 \%$ & \\
\hline \multicolumn{5}{|l|}{ Data collection } \\
\hline Length of data collection (weeks) & 47 & 52 & 55 & 53 \\
\hline Pre CMF & 11 & 18 & 18 & 20 \\
\hline Post CMF & 36 & 34 & 38 & 33 \\
\hline Number of conflicts Identified ( $n$ ) & 432 & 216 & 147 & 205 \\
\hline Pre CMF & 76 & 120 & 45 & 87 \\
\hline Post CMF & 356 & 96 & 102 & 118 \\
\hline
\end{tabular}

Site D had undergone training in conflict management previously, so the training was limited to one half-day focused on application of the CMF tool.

*Senior nurses include sisters, paediatric and advanced nurse practitioners and clinical nurse specialists.

†Therapists include occupational therapists, physiotherapists, dieticians, speech and language therapists and play therapists.

$\mathrm{CMF}$, conflict management framework.

undertaken to implement and evaluate the impact of the CMF on conflict within four tertiary UK paediatric services.

\section{METHODS}

\section{Sites}

Four sites were included in the intervention and evaluation: Evelina London Children's Hospital, Leeds Children's Hospital, the Royal Marsden Hospital Children and Young People's Unit and Southampton Children's Hospital. Collectively, these sites included eight clinical areas across medical, surgical and intensive care specialties.

In preparation for the introduction of the CMF protocol, staff within the four sites attended conflict management training workshops run by MMF. Workshops focused on three themes: conflict identification, conflict management skills and use of the CMF to provide structure to conflict identification and management across clinical teams.

Overall, 251 staff members attended a total of 15 full-day training sessions across three of the sites (see table 1). The fourth site (site D) had been conducting staff training in conflict management for the previous 5 years, so at this site a half-day training session focused on implementing the CMF was delivered to 14 senior nursing staff. Site C adopted a quality improvement approach for the implementation of the CMF, using run charts to inform staff on monthly progress.

\section{Intervention}

After preintervention training, each site implemented the CMF on a set date as a component of existing daily huddles and handovers (see figure 1). The CMF flowcharts (see online supplemental material 1) were used to prompt staff on the appropriate actions to take to de-escalate conflict. Actions taken were recorded on conflict data collection forms.

\section{Conflict management framework}

The CMF is a two-stage framework that provides a structure for clinicians to implement conflict management into routine practice (see online supplemental material 1). 


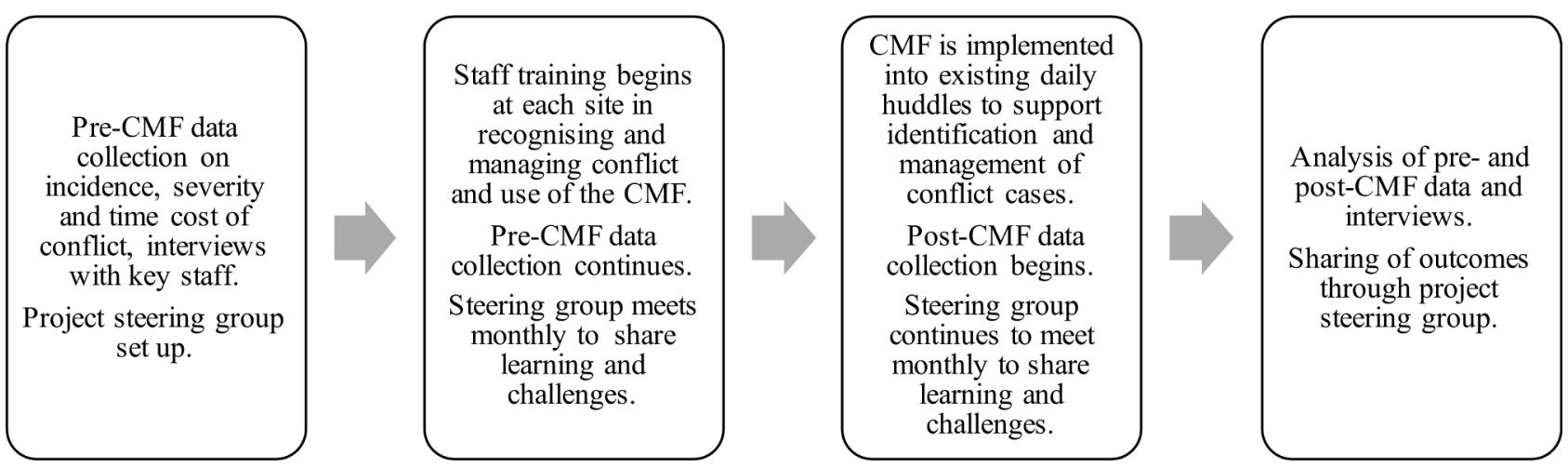

Figure 1 Data collection, implementation and oversight of the conflict management framework (CMF).

CMF stage one is incorporated as a routine component of daily huddles or handovers and staff members review whether there are early signs of conflict around the care of any patient. Where signs of conflict are identified, the framework provides a structure for staff to engage with the family as soon and as effectively as possible, using the skills of compassionate communication and mediation skills from the preintervention training. Engagement at stage one involves identifying the staff members who will engage with the family to explore their concerns, establish underlying causes of the conflict and agree on a de-escalation plan. If stage one is unable to resolve the conflict and concerns are raised that the conflict could impact the quality of care of a patient, CMF stage two is activated.

CMF stage two is a more formal process of conflict management arranged in a series of steps for systematic and timely progression of actions, involving safeguarding legal, mediation and ethics teams when appropriate. The process begins with a collaborative 'acceptance of responsibilities agreement' between family and staff, escalates through formal warnings and if needed, ends with the exclusion of family member(s) from the service. A senior health professional takes responsibility for ensuring appropriate actions are followed and key senior staff at clinical and management levels are kept informed. The stepwise progression in stage two aims to empower and prompt staff to act collaboratively to reduce the likelihood of progression to formal warning or exclusion.

\section{Evaluation}

Conflict data collection

Quantitative data regarding conflicts were collected by senior ward nurses at each site. Preintervention and postintervention data collection sheets recorded time spent managing conflict, conflict severity and the staff members involved (see online supplemental material 2 ). Time spent managing conflict is one of the impacts of conflict most frequently reported by staff ${ }^{516}$ and was therefore selected as a main impact metric along with conflict severity and financial cost.

Baseline data were collected prospectively for 11-20 weeks before adoption of the CMF tool (see table 1 for breakdown by site). These data were collected by staff who had completed their conflict management training, to help prevent response-shift bias ${ }^{17}$ after training. Postintervention data were collected for 33-38 weeks (see table 1) and included the CMF actions taken (see online supplemental material 2).

\section{Data analysis}

Using the statistical package SPSS, we fitted median regression models for time spent on conflict, cost and maximum conflict severity score reached with each patient/family (the outcome variables). The independent (explanatory) variables for both models included timing of the conflict (before or after implementation of the CMF), hospital site, age of patient and the number of patients on the ward at the time of conflict. Conflict severity was included as an additional independent variable for the models for time and cost. Beta coefficients describing the relationship between the explanatory variables and the outcome variables were estimated by simplex algorithm. Alpha was set at the 0.05 level.

Cost of conflict was conservatively calculated using the hourly rates associated with the lowest pay point for each staff type category.

Where conflict cases crossed the implementation date, maximum conflict severity score for that conflict case was reported as pre or post CMF based on the date that maximum score was reached.

\section{Interviews and interview analysis}

Semistructured interview questions focused on staff objectives for the CMF, their experiences of the implementation and local adaptation of the CMF and the perceived impact of the CMF in their site. Purposive sampling was used to identify interviewees who had a lead role for implementing or facilitating use of the CMF and therefore were well placed to offer rich accounts of their experiences and challenges implementing the CMF.

A total of 16 interviews were conducted with 11 interviewees by a female researcher (LF) with a $\mathrm{PhD}$ in health research and experience of healthcare conflict research. Interviews were conducted pre intervention $(n=5)$, midintervention $(\mathrm{n}=5)$ and post intervention $(\mathrm{n}=6)$. One doctor and senior nurse requested to be interviewed as a dyad at all three timepoints. Interviews lasted up to 
$45 \mathrm{~min}$ and were recorded for full transcription, with recorded verbal consent.

Qualitative data were coded and analysed by LF using a five-step process: familiarisation with the data set; identifying a thematic framework; indexing the data with reference to the thematic framework; synthesising responses into a working grid of themes and data interpretation and finalisation of key themes. Saturation of core themes was reached within the sample interviewed.

\section{Governance}

This project was reviewed by hospital clinical governance bodies at all four sites. As an evaluation of a staff development programme, ethics approval was not required.

A steering group comprised of senior medical and nursing clinicians from each site, academic evaluators and the conflict training leads met monthly.

\section{Patient and public involvement}

Patients were involved in the design and development of the CMF. Families of children who had experienced conflict were approached both formally and informally for their input into the framework. The questions we sought to address in the pilot project were developed in consultation with patients, healthcare staff and organisational stakeholders and outcome measures were selected for their relevance to these groups. Staff leads from each site were invited to formally join the project team, to share their learning throughout the project and to disseminate learning through their local channels.

\section{RESULTS}

\section{Conflicts}

Conflicts were recorded for a total of 67 weeks before and 141 weeks after implementation of the CMF across the four sites. There were 1000 episodes of conflict recorded with 324 patients/families across the eight included clinical areas. The median patient age was 12 months (IQR 3 months -9 years, $\mathrm{n}=328$ ) in conflicts before implementation and 24 months (IQR 3 months-13 years, n=672) after implementation. Median patient age varied across sites, as shown in table 1.

\section{Quantitative results}

The median regression models are shown in online supplemental material 3. Time spent managing episodes of conflict with a patient was decreased after the CMF implementation by $24 \% \quad(\mathrm{p}<0.001) \quad$ (from $73 \mathrm{~min}$ to $55 \mathrm{~min}$ ) and the cost of this staff time decreased by $20 \%$ ( $p<0.02$ ) (from $£ 26$ to $£ 21$ sterling per episode of conflict). The maximum conflict severity reached with each conflict case did not significantly change after application of the CMF.

Time and cost of conflict was higher when the conflict cases related to older patients and in cases with more severe conflict. While the number of patients on a ward did not have a significant effect on time or cost of conflict,
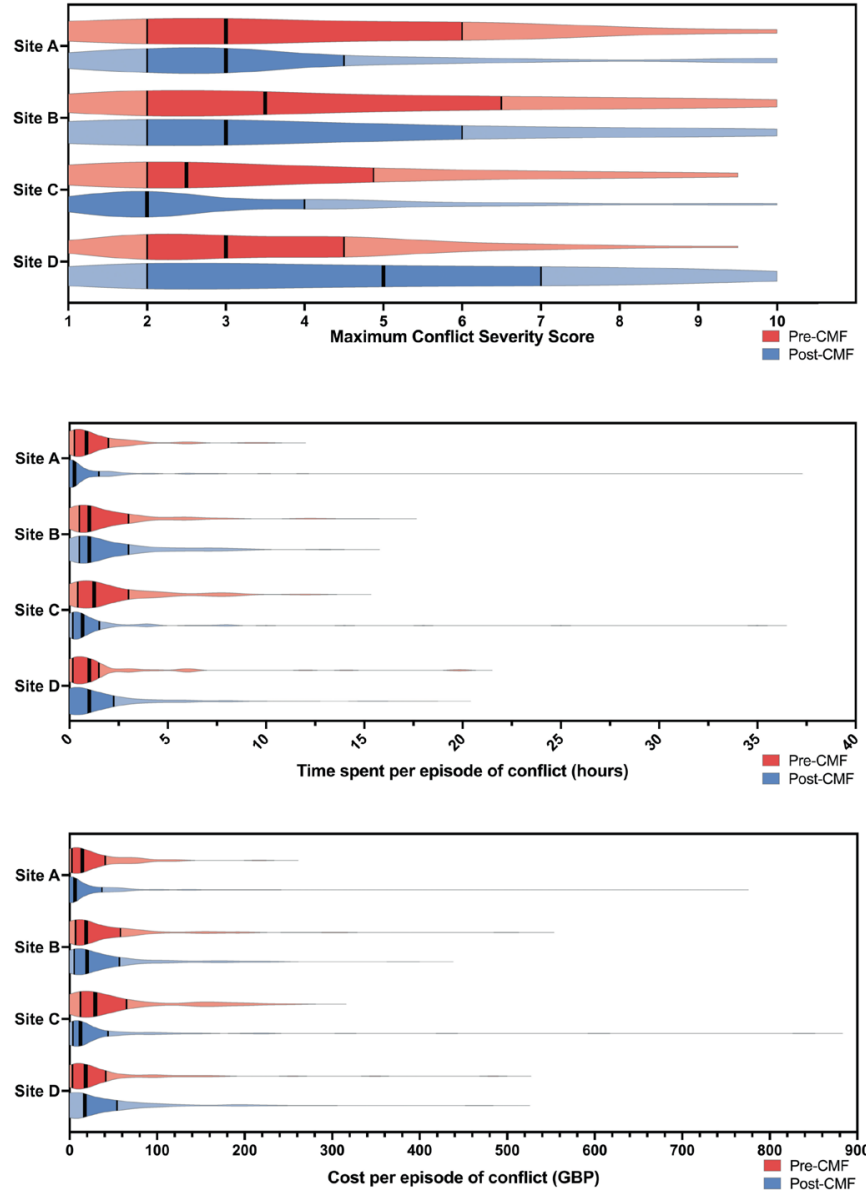

Figure 2 Violin plots for each site for severity, time and cost of conflict, pre CMF (red) and post CMF (blue) implementation. Vertical lines within each violin plot represent the median (thicker vertical line) and quartiles (thinner lines) for each site. Interquartile ranges are shaded darker red/ blue. These violin plots provide additional information about conflict at each site compared with box-and-whisker plots, with the width of each violin plot indicating the number of conflict episodes clustering at each time or cost value. CMF, conflict management framework. GBP, pounds sterling.

higher number of patients was associated with increased clinician-rated conflict severity scores.

Variation in time, cost and severity of conflicts was noted between sites, as shown in the median regression analysis (online supplemental material 3) and in figure 2. In particular, site A was noted to have reported lower impact of conflict in all three measures. At all sites, the maximum time spent on certain single episode of conflict at each site was many times higher than the median time (see figure 2). These extreme time costs equated to equally extreme financial costs for staff time. At site $\mathrm{D}$, where conflict resolution training had been delivered over the preceding 5 years, higher severity of conflict was recorded both pre and post intervention, however the time staff spent to manage this conflict reduced significantly after implementation of the CMF tool. 


\section{Qualitative results}

Themes generated from the interviews related to a challenge to the prevailing culture, the need for crossdisciplinary leadership and the need for conflict management training alongside the CMF.

\section{A challenge to the prevailing culture}

Using the CMF resulted in a fundamental culture change in both naming and then addressing behaviours which have traditionally been accommodated and normalised.

It's a real, real culture change from the way we've been brought up in the NHS of ... it's natural families complain, you just have to kind of crack on and deal with and appease them and ... just simmer things down quite quickly, rather than unpicking it more and letting ... and opening it up. (Doctor, site $\mathrm{D}$, mid-intervention)

Some staff felt that using the framework led to discomfort, requiring them to confront families and move out of their comfort zones.

It took all my courage to actually do the 'responsibility agreement' with this family. Everything in me wanted to duck out of that. I did do it. (Doctor, site $\mathrm{B}$, mid-intervention)

Interviewees suggested that it was more effective when senior staff members lead conversations with families about conflict.

You have got this need to maintain a therapeutic relationship, and if the person delivering that message is part of the day to day clinical team, that sets up a kind of conflict in itself [...] I would be a strong advocate that when it gets to a stage two, for everyone's benefit you have someone that's outside of the clinical team that can be seen as impartial. (Nurse, site D, post intervention)

\section{The need for cross-disciplinary leadership}

Interviewees at all sites viewed strong leadership from both nurses and doctors to be necessary for effective implementation of the GMF.

You need at least one champion in each area. So you need a consultant, you need a junior doctor, and you need a nurse and then senior nurse or matron.... I think you listen to people much more when you're a similar kind, and also they understand your challenges. (Doctor, site D, mid-intervention)

They felt that this leadership needed to be from champions in the wards implementing the CMF, rather than more distally located, to ensure conflict was considered daily.
We've got a few champions on the unit... making sure it's mentioned... So it has just been more, you know, it has been more visible to see really. (Nurse, site A, post intervention)

\section{The CMF complemented conflict training}

All interviewees reported training on conflict signs and management strategies was integral to use of the CMF and more important than training on use of the framework alone.

[The training is] amazing, and the challenging, the language that you use. I put my team through it recently. There were some people that hadn't done it and felt quite strongly that they needed to. And they came out saying 'oh my god, it's just amazing. I've completely changed how I speak to parents.' (Nurse, site $\mathrm{D}$, post intervention)

The CMF itself was seen to complement training by guiding how staff de-escalated conflict.

I don't think training alone [is enough] because I think there has to be a process with trigger points, which the framework provides. (Doctor, site C, midintervention)

\section{The number of staff trained varied between sites}

The number of staff trained in site $\mathrm{A}$ and site $\mathrm{B}$ was considerably higher than in site $\mathrm{C}$ and site D (see table 1). Sites A and B both had a senior medical and nursing lead and dedicated administrative input in organising the training sessions and booking staff to attend. In contrast, site $\mathrm{C}$ had two senior medical leads but no additional administrative support to organise staff to attend the training and no nursing lead.

\section{DISCUSSION}

This pilot project demonstrated a reduction in staff time spent managing conflict and the associated staff cost of managing conflict after the implementation of the structured CMF and associated training. This reduction in time and cost occurred despite conflict episodes having similar severity after implementation of the CMF. Interviews highlighted that use of the $\mathrm{CMF}$ provoked a significant challenge to the prevailing culture of conflict avoidance. Interviewees stressed the importance of crossdisciplinary leadership to champion the introduction of the framework and the need to combine conflict management training with use of the CMF.

In line with previous work suggesting that a considerable proportion of time and economic cost of conflict result from a small number of highly fractious conflicts, ${ }^{2111819}$ each site had a small number of conflict 
cases that required extreme amounts of staff time both inside and outside the clinical team.

There was significant variation in the impact of conflict reported between the sites. It is possible that some of the variation resulted from wider training at site $\mathrm{A}$ and site B compared with site $\mathrm{C}$ and site $\mathrm{D}$. This would be in line with comments from interviewees about the importance of local champions for successful implementation of the CMF. The age range for patients in site B was older (1-24 years) than the other three sites $(0-17$ years). Increased time and financial cost associated with managing episodes of conflict associated with the care of older patients may have obscured the impact of the widespread training at site B. Further comparative research could focus on comparison between sites and identification of possible reasons for variation in impact of the CMF.

\section{Limitations}

The data used in this evaluation relied on clinician ratings, which would have been subject to some degree of inter-rater variation. We attempted to limit this variability by including a graded scale on the data collection sheet for conflict severity (online supplemental material 2), by ensuring that data collection sheets recording severity and time spent were filled in daily and by formally training designated individuals involved in data collection before they collected preCMF or post-CMF data.

Our assessment of the staff cost of conflict was deliberately conservative, based on the lowest salary point of a given staff type. We did not include other direct costs (such as legal costs for cases which were escalated beyond the clinical team, loss of staff productivity, staff absences or turnover, increased patient lengths of stay) or indirect costs of conflict (such as staff psychological distress). As such, the financial cost of conflict pre and post implementation of the CMF is likely to be considerably understated, as are the time costs.

There was considerable variation in the number of staff trained at each site, which could have limited the impact of the CMF. In the light of the positive impact of the CMF, further research is warranted into factors which affect the effectiveness of the framework.

The interviews included in this study were with staff members closely involved in the implementation of the CMF at each of the sites. These interviewees were well placed to comment on the experience and challenges of implementation, as they had a rich overview of the project from the perspective of their respective sites.

\section{CONCLUSION}

This multisite pilot project suggests that the CMF shows promise as a tool for supporting staff to manage healthcare conflict, reducing time spent managing conflict and the associated staff costs. Qualitative findings highlighted the value of having a formal structure with which to identify and manage conflict at different levels and the importance of multidisciplinary leadership to help embed a consistent approach. Providing clinicians with the skills and confidence to address conflict before it escalates may also combat a tendency by health professionals to avoid and tolerate conflict. This is likely to lead to improved staff wellbeing and greater patient/family satisfaction with the care and communication they receive from those caring for them or their child. Further testing of the CMF is required to evaluate its impact in additional sites over an extended period of time, including possible impact for staff. This work has been funded and began in Spring 2021.

\section{Author affiliations}

${ }^{1}$ Nuffield Department of Surgical Sciences, Oxford University, Oxford, UK

${ }^{2}$ Faculty of Social Sciences, University of Stirling, Stirling, UK

${ }^{3}$ Medical Mediation Foundation, London, UK

${ }^{4}$ Paediatric Oncology, Royal Marsden Hospital NHS Trust, London, UK

${ }^{5}$ Paediatrics, University Hospital Southampton NHS Foundation Trust, Southampton, UK

${ }^{6}$ Clarendon Wing, Leeds Children's Hospital, Leeds, UK

${ }^{7}$ Paediatrics, Evelina London Children's Hospital, London, UK

${ }^{8}$ Population Health Sciences, Bristol Medical School, Bristol, UK

${ }^{9}$ Whipps Cross University Hospital, London, UK

${ }^{10}$ Children and Young People's Unit, Royal Marsden Hospital NHS Trust, London, UK

${ }^{11}$ Paediatrics, Southampton Children's Hospital, Southampton, UK

Acknowledgements The authors would like to thank Clare Hazlegreaves from Leeds Children's Hospital for her significant contributions to this project and Helena Robson from the Evelina London Children's Hospital and Kirsty Easton from the Royal Marsden Hospital for their contributions to collecting and entering data. The authors would also like to thank Dr Jonathan Ives, University of Bristol, for his contribution to the data analysis and conclusions of the paper.

Contributors LF and SB designed the work. LF, CSnowball, JCC, NT, HR, CH and $\mathrm{SC}$ acquired the data. CSayer designed the databases. LF, OL, JCC, EM, KP, SI and SB interpreted the data, drafted the work and revised it critically for intellectual content. All authors approved the final version of the manuscript and agreed to be accountable for all aspects of the work, ensuring that questions related to the accuracy or integrity of any part of the work are appropriately investigated and resolved. $\mathrm{OL}$ acts as primary guarantor.

Funding JCC was supported by the Royal Marsden Cancer Charity and by National Health Service funding to the National Institute for Health Research Biomedical Research Centre of The Royal Marsden Hospital. Leeds Cares hospital charity funded the costs of the conflict management training at Leeds Children's Hospital. Each site contributed to the cost of evaluating the pilot project and funded the conflict management training, either directly or through their hospital charities.

Competing interests SB is a director of the Medical Mediation Foundation (MMF), an organisation that provides conflict management training and mediation to resolve disagreement/conflict between patients, families and healthcare professionals. EM is an associate of the MMF.

Patient consent for publication Not applicable.

Ethics approval As an evaluation of a staff development programme, ethics approval was not required.

Provenance and peer review Not commissioned; externally peer reviewed.

Data availability statement Data are available upon reasonable request. Data are available on request.

Supplemental material This content has been supplied by the author(s). It has not been vetted by BMJ Publishing Group Limited (BMJ) and may not have been peer-reviewed. Any opinions or recommendations discussed are solely those of the author(s) and are not endorsed by BMJ. BMJ disclaims all liability and responsibility arising from any reliance placed on the content. Where the content 
includes any translated material, BMJ does not warrant the accuracy and reliability of the translations (including but not limited to local regulations, clinical guidelines, terminology, drug names and drug dosages), and is not responsible for any error and/or omissions arising from translation and adaptation or otherwise.

Open access This is an open access article distributed in accordance with the Creative Commons Attribution Non Commercial (CC BY-NC 4.0) license, which permits others to distribute, remix, adapt, build upon this work non-commercially, and license their derivative works on different terms, provided the original work is properly cited, appropriate credit is given, any changes made indicated, and the use is non-commercial. See: http://creativecommons.org/licenses/by-nc/4.0/.

\section{ORCID iDs}

Oscar Lyons http://orcid.org/0000-0001-5809-7173

Liz Forbat http://orcid.org/0000-0002-7218-5775

Kate Pryde http://orcid.org/0000-0001-9679-8018

\section{REFERENCES}

1 Almost J, Wolff AC, Stewart-Pyne A, et al. Managing and mitigating conflict in healthcare teams: an integrative review. J Adv Nurs 2016;72:1490-505.

2 Shin J-H. Developing constructive and proactive conflict management strategies in healthcare. $J$ Commun Healthc 2009;2:78-94.

3 Haraway DL, Haraway WM. Analysis of the effect of conflictmanagement and resolution training on employee stress at a healthcare organization. Hosp Top 2005;83:11-17.

4 Brinkert R. A literature review of conflict communication causes, costs, benefits and interventions in nursing. J Nurs Manag 2010;18:145-56.

5 Gerardi D. Using mediation techniques to manage conflict and create healthy work environments. AACN Clin Issues 2004;15:182-95.

6 Rowe MM, Sherlock $\mathrm{H}$. Stress and verbal abuse in nursing: do burned out nurses eat their young? J Nurs Manag 2005;13:242-8.
7 Saulo M, Wagener RJ. Mediation training enhances conflict management by healthcare personnel. Am J Manag Care 2000;6:473-83.

8 Forbat L, Teuten B, Barclay S. Conflict escalation in paediatric services: findings from a qualitative study. Arch Dis Child 2015;100:769-73.

9 Almost J. Conflict within nursing work environments: concept analysis. John Wiley \& Sons, Ltd, 2006.

10 Forbat L, Simons J, Sayer C, et al. Training paediatric healthcare staff in recognising, understanding and managing conflict with patients and families: findings from a survey on immediate and 6-month impact. Arch Dis Child 2017;102:250-4.

11 Wallis $\mathrm{C}$. When paediatricians and families can't agree. Arch Dis Child 2018;103:413-4.

12 Jones L. The application of organisational conflict management: a mixed method exploration of conflict training and perceptions of NHS managers, 2016.

13 Sullivan $\mathrm{AH}$. A qualitative study of the perceived impact of management training in conflict resolution in a hospital. 3171573 2005.

14 Forbat L, Barclay S. Reducing healthcare conflict: outcomes from using the conflict management framework. Arch Dis Child 2019;104:328-32.

15 The Medical Mediation Foundation. Resolving conflicts in health and social care.

16 Forbat L, Sayer C, McNamee P, et al. Conflict in a paediatric Hospital: a prospective mixed-method study. Arch Dis Child 2016;101:23-7.

17 Howard GS, Dailey PR. Response-shift bias: a source of contamination of self-report measures. J Appl Psychol 1979;64:144-50.

18 Wilkinson D, Barclay S, Savulescu J. Disagreement, mediation, arbitration: resolving disputes about medical treatment. Lancet 2018;391:2302-5.

19 Wilkinson D, Savulescu J, Evans A. and Charlie Gard - Should the law change? BMJ 2018;361. 